\title{
Hyperglycemia Suppresses RANKL-Induced Osteoclast Differentiation through LXR $\beta$ Expression in RAW264.7 Cells
}

\author{
Teruyoshi TANAKA ${ }^{1,2}$, Yuichiro TAKEI ${ }^{1,3}$, Nobuhiro ZAIMA ${ }^{2}$, \\ Tatsuya MORIYAMA ${ }^{2}$ and Dai YAMANOUCHI ${ }^{1, *}$ \\ ${ }^{1}$ Division of Vascular Surgery, Department of Surgery, University of Wisconsin School of Medicine and \\ Public Health, 1111 Highland Ave. 5148 Wisconsin Institute for Medical Research, \\ Madison, WI 53705, USA \\ ${ }^{2}$ Department of Applied Biological Chemistry, Graduate School of Agriculture, \\ Kindai University, 3327-204, Naka-machi, Nara 631-8505, Japan \\ ${ }^{3}$ Faculty of Nutrition, University of Kochi, 2751-1 Ike, Kochi, Kochi 781-8515, Japan
}

(Received July 4, 2016)

\begin{abstract}
Summary There have been reports that hyperglycemia suppresses osteoclast (OCL) differentiation, although the underlying mechanism is poorly understood. Here we demonstrated that high glucose suppresses OCL differentiation through activation of liver X receptor (LXR) $\beta$, a recently reported glucose-sensing nuclear receptor. The effect of hyperglycemia on osteoclastogenesis was tested in RAW264.7 cells, a murine macrophage cell line. Cells were treated with receptor activator of NF- $\kappa$ B ligand (RANKL) under normoglycemic $(5.5 \mathrm{~mm}$ glucose), normoglycemic with high osmotic pressure $(5.5 \mathrm{~mm}$ glucose $+10.0 \mathrm{~mm}$ mannitol), and hyperglycemic (15.5 mM glucose) conditions. RANKL-induced osteoclastogenesis was significantly suppressed by high-glucose treatment. Mannitol treatment also significantly suppressed osteoclastogenesis, but the inhibitory effect was lower than for high-glucose treatment. The suppression of mRNA expression of $\operatorname{Lxr} \beta$ by RANKL was significantly restored by high glucose, but not mannitol. Additionally, the deactivation of $\operatorname{Lxr} \beta$ by siRNA attenuated high-glucose-induced suppression of osteoclastogenesis. Although further validation of the underlying pathway is necessary, $\operatorname{targeting} \operatorname{LXR} \beta$ is a potential therapeutic approach to treating osteoporosis.
\end{abstract}

Key Words osteoclast differentiation, hyperglycemia, liver X receptor $\beta, \operatorname{LXR} \beta$

Osteoclasts (OCLs), derived from the monocyte/macrophage cell lineage, contribute to bone resorption (1-3). It is well reported that nuclear factor $\kappa-\mathrm{B}(\mathrm{NF}-\kappa \mathrm{B})$, receptor activator for nuclear factor $\kappa$-B ligand (RANKL), osteopontin, parathyroid hormone, macrophage colony stimulating factor (M-CSF), and angiotensin-II all play important roles in osteoclastogenesis (1-3). Monocytes/ macrophages induced by these factors first proliferate actively and become committed preosteoclasts, tartrateresistant acid phosphatase (TRAP)-positive mononuclear cells, which then fuse to one another to become mature multinucleated OCLs $(1,4,5)$. These cells secrete $\mathrm{HCl}$ to dissolve bone into its calcium salts $(1,6)$.

For bone degradation, glucose is a principal energy source (7), and therefore blood glucose concentration affects OCL differentiation. For example, glucose at 0.5-5 mM dose-dependently increased TRAP activity, while $40 \mathrm{~mm}$ glucose treatment suppressed OCL differentiation (8). Wittrant et al. reported that $25 \mathrm{~mm} \mathrm{D-glu-}$ cose treatment suppressed RANKL-induced osteoclastogenesis (9) and suggested that high-glucose treatment for $6 \mathrm{~d}$ exerts an inhibitory action on NF- $\kappa \mathrm{B}$, which is an oxidative stress marker, by suppressing production

\footnotetext{
*To whom correspondence should be addressed.

E-mail: yamano@surgery.wisc.edu
}

of reactive oxygen species. Although these reports suggested that hyperglycemia suppresses osteoclastogenesis in vitro, the glucose concentrations they used are too high and not relevant to hyperglycemia seen in humans in vivo, even in diabetes patients. Krinsley (10) reported hospital mortality increased progressively as glucose values increased, reaching $42.5 \%$ among patients with mean glucose values exceeding $300 \mathrm{mg} / \mathrm{dL}(16.7 \mathrm{mM})$. Thus, further studies are required with the appropriate glucose concentrations less than or equal to $16.7 \mathrm{mM}$, which mimic mild diabetes patients.

In addition, type 1 diabetes mellitus (DM) is clinically associated with decreased bone mineral density (11, 12) by increasing OCL activity (13), and Catalfamo et al. demonstrated that osteoclastogenesis was not suppressed by LPS treatment in bone marrow-derived macrophages from type $2 \mathrm{DM}$ patients and mice (14). On the other hand, bone mineral density is increased in type 2 DM patients (11). Type $1 \mathrm{DM}$ is developed by offset of insulin production in pancreatic $\beta$ cells. Type $2 \mathrm{DM}$ is characterized by insulin resistance, impaired insulin secretion, and increased glucose concentration. Because there is the difference in osteoclastogenesis between type 1 (insulin depletion) and 2 (insulin resistance) DM, further studies are needed for clarifying the effect of hyperglycemia on RANKL-induced osteoclast differen- 
tiation in vitro.

Liver $\mathrm{X}$ receptor (LXR) $\alpha$ and $\beta$ are nuclear receptors that have recently been reported to be activated by D-glucose, D-glucose-6-phosphate, and the LXR agonist GW3965 (15). There has also been great interest in these receptors as novel therapeutic targets for inflammatory diseases, as they inhibit inflammatory gene expression downstream of toll-like receptor 4-, interleukin-1 $\beta$-, and tumor necrosis factor $\alpha$-mediated signaling $(16,17)$. Robertson Remen et al. demonstrated the effect of GW3965 in lipopolysaccharide (LPS) with RANKL-induced differentiation of bone marrow-derived macrophages (BMMs) (18) and RANKL-induced differentiation of RAW264.7 cells, a murine macrophage cell line (19), into OCLs via an LXR $\beta$-dependent (but not an LXR $\alpha$-dependent) mechanism.

Therefore, in this study BMMs or cells of the murine monocyte/macrophage cell line RAW264.7 were treated with $15.5 \mathrm{~mm}(279 \mathrm{mg} / \mathrm{dL})$ glucose to examine how high glucose affects OCL differentiation through LXR $\beta$.

\section{MATERIALS AND METHODS}

Materials. All chemicals used in this study were of the highest purity available.

Cell culture and treatment. All animal procedures were conducted in accordance with experimental protocols that were approved by the Institutional Animal Care and Use Committee at the University of Wisconsin, Madison (Protocol M02394). Primary monocytes/ macrophages were obtained from 4- to 6-wk-old male mouse bone marrow and cultured in minimal essential medium (MEM)- $\alpha$ with 10\% fetal bovine serum (FBS), $100 \mathrm{IU} / \mathrm{mL}$ penicillin, $100 \mu \mathrm{g} / \mathrm{mL}$ streptomycin, and $20 \mathrm{ng} / \mathrm{mL}$ recombinant mouse M-CSF. BMMs were isolated using Ficoll-Paque medium (GE Healthcare, Piscataway, NJ) and pre-cultured in MEM- $\alpha$ overnight. One day later, non-adhesive cells were collected and replated into 96-well plates at 3,000 cells per well. The BMMs were then treated with $100 \mathrm{ng} / \mathrm{mL}$ recombinant murine RANKL (Peprotech, Rocky Hill, NJ) and $40 \mathrm{ng} / \mathrm{mL}$ M-CSF. Murine monocytic RAW264.7 cells were purchased from American Type Culture Collection (Manassas, VA) and maintained in Dulbecco's modified Eagle's medium (DMEM) containing 10\% FBS, $100 \mathrm{IU} / \mathrm{mL}$ penicillin, and $100 \mu \mathrm{g} / \mathrm{mL}$ streptomycin. For OCL differentiation analysis, 3,000 cells were plated in each well of a 96-well plate and maintained in MEM- $\alpha$ medium supplemented with $10 \%$ charcoal-stripped FBS with antibiotics. For protein analysis, $3 \times 10^{5}$ cells were plated per well in a 6 -well plate. The cells were then stimulated with $50 \mathrm{ng} / \mathrm{mL}$ RANKL, which was added on alternate days to the cultures. Five days after the treatment, the cells were fixed with 4\% paraformaldehyde (PFA) and then stained using the TRAP staining method. OCLs were identified as TRAP-positive, multinucleated $(>3$ nuclei) cells and scored in each well.

siRNA transfection. RAW 264.7 cells were transfected with either a predesigned LXR $\beta$ siRNA (Invitrogen, Carlsbad, CA) or Silencer Negative Control \#1 siRNA (Invitrogen) using Lipofectamine RNAiMAX transfec- tion reagent (Invitrogen), according to the manufacturer's recommendations. Transfected cells $\left(3 \times 10^{5}\right)$ were seeded in 6-well plates for mRNA extraction.

Cell viability. RAW 264.7 cells (3,000 cells per well) were cultivated in 96-well plates $1 \mathrm{~d}$ before treatment with RANKL, mannitol, and glucose. The cells were supplemented with MTT solution [3-(4,5-dimethyl-thiazol2-yl)-2,5-diphenyltetrazolium bromide (50 ng/well)] and incubated for $4 \mathrm{~h}$. Acid-isopropanol $(0.04 \mathrm{~N} \mathrm{HCl}$ in isopropanol) and 3\% sodium lauryl sulfate were added to dissolve the reduced MTT crystals (formazan) present in the cells. After mixing, the absorbance was measured at $595 \mathrm{~nm}$ by a microplate reader, using $655 \mathrm{~nm}$ for the reference.

Total protein and RNA recovery. Protein extraction was performed at $0-4^{\circ} \mathrm{C}$. Total protein was extracted with a radioimmunoprecipitation assay buffer with protease inhibitor cocktail (Cell Signaling Technology, Danvers, MA). Protein contents were determined by using a DC Protein Assay Kit II (Bio-Rad, Hercules, CA). Bovine serum albumin was used as a standard protein. Total RNA was extracted from the RAW264.7 cells using a TRIzol Plus RNA Purification Kit (Invitrogen), according to the manufacturer's instructions. RNA concentration was estimated by measuring the absorbance at $260 \mathrm{~nm}$.

Western blotting. Twenty-microgram samples were separated on $8-12 \%$ polyacrylamide gels in Laemmli buffer and transferred electrophoretically onto polyvinylidene difluoride membranes. The primary antibodies used for Western blotting included mouse NFATc1 (sc7294, Santa Cruz Biotechnology, CA), rabbit LXR $\beta$ (sc1001, Santa Cruz Biotechnology), and mouse $\alpha$-tubulin (sc-23948, Santa Cruz Biotechnology) antibodies. Primary antibodies were detected using a horseradish peroxidase-conjugated secondary antibody and visualized with the Enhanced Chemiluminescence kit (Thermo Scientific, Rockford, IL) or SuperSignal West Femto Maximum Sensitivity Substrate (Thermo Scientific).

Quantitative real-time PCR. Total RNA was extracted from the RAW264.7 cells using a TRIzol Plus RNA Purification Kit (Invitrogen), according to the manufacturer's instructions. Quantitative real-time PCR was performed with SYBR Green dye in a real-time PCR instrument (ABI, Foster City, CA). Mouse Gapdh primers for quantitative RT-PCR were purchased from Qiagen (\#PPM02946E, Valencia, CA). The sequences of other primers used were as follows: Mmp9 F, 5'-CATTCGCGTGGATAAGGAGT-3', and R, 5'-GTTCACCTCATGGTCCACCT-3'; Ctsk F, 5'-CGAAAAGAGCCTAGCGAACA-3', and R, 5'-TGGGTAGCAGCAGAAACTTG-3'; Nr1h2 (also known as $\operatorname{Lxr} \beta$ ) F, 5'-CAGCAGCTCAGGCCGGCAG-3', and R, 5'-GGCTAGCTCGGTGAAGTGGG-3'. The expression level for each gene was normalized to the Gapdh expression level in the same sample.

Statistical analysis. Data are reported as mean \pm SD. Statistical analysis was performed with the GraphPad Prism program, version 4.00 (GraphPad Software, Inc., San Diego, CA). For testing 2 samples from the same population (Fig. 2A), the Wilcoxon rank sum test was used. Differences for Fig. 3A was analyzed with a $t$-test. 
Table 1. Effect of high glucose treatment on cell viability, numbers of cell recovery, and total protein and RNA recovery.

\begin{tabular}{|c|c|c|c|c|}
\hline & NT & RANKL & Man & H-Glc \\
\hline Cell viability (\%) & $100.0 \pm 2.1$ & $101.7 \pm 3.8$ & $101.3 \pm 4.9$ & $105.7 \pm 5.1$ \\
\hline Numbers of cell $\left(\times 10^{5}\right)$ & $3.4 \pm 0.3$ & $3.3 \pm 0.2$ & $3.5 \pm 0.1$ & $3.9 \pm 0.3$ \\
\hline Protein $(\mu \mathrm{g})$ & $91.3 \pm 9.0$ & $127.0 \pm 11.3$ & $126.7 \pm 11.5$ & $101.3 \pm 22.0$ \\
\hline $\mathrm{RNA}(\mu \mathrm{g})$ & $0.4 \pm 0.0$ & $0.6 \pm 0.1^{*}$ & $0.6 \pm 0.0^{*}$ & $0.5 \pm 0.0$ \\
\hline
\end{tabular}

RAW264.7 cells were cultured for $2 \mathrm{~d}$ under normoglycemic conditions (N-Glc, $5.5 \mathrm{~mm}$ glucose) without RANKL (no treatment [NT] control), N-Glc with RANKL, normoglycemic conditions with high osmotic pressure (Man, 5.5 mM glucose and $10.0 \mathrm{~mm}$ mannitol) and RANKL, and hyperglycemic conditions (H-Glc, $15.5 \mathrm{~mm}$ glucose) with RANKL. Values are presented as means \pm SD for at least three replicates. ${ }^{*} p<0.05$, vs. NT.

A

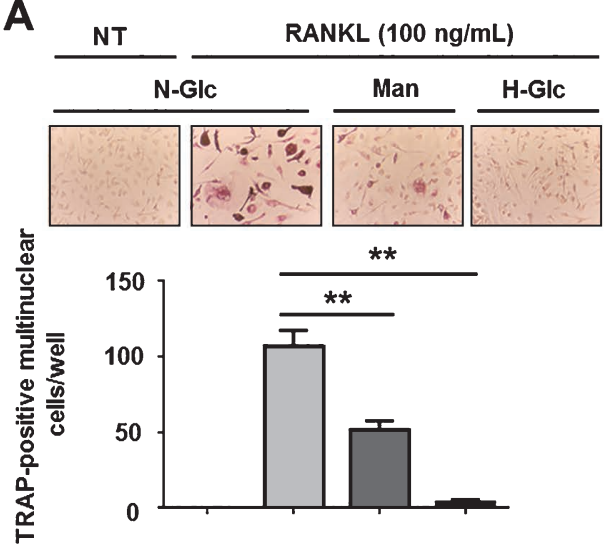

B
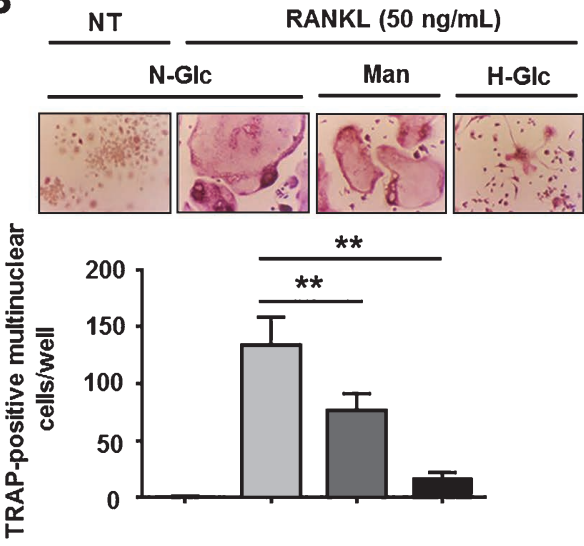

C

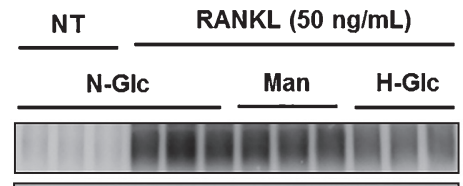

Tubulin

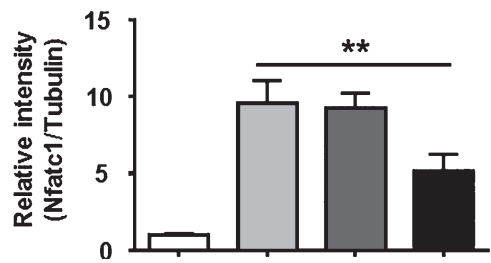

D

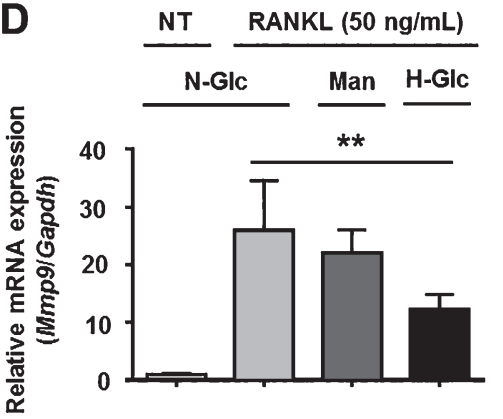

$\mathbf{E}$

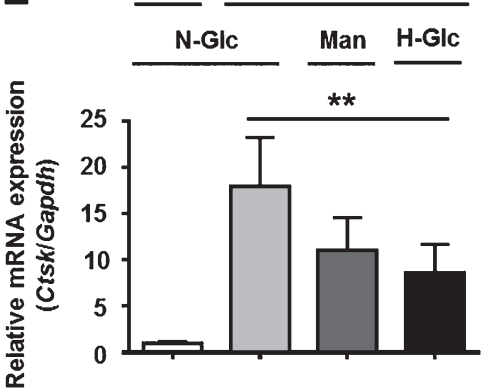

RANKL (50 ng/mL)

Fig. 1. High-glucose treatment suppresses RANKL-induced OCL differentiation. Representative images of TRAP staining and the numbers of TRAP-positive cells. Primary monocytes/macrophages derived from murine bone marrow (A) and RAW264.7 cells (B) were cultured for $5 \mathrm{~d}$ under normoglycemic conditions (N-Glc, $5.5 \mathrm{~mm}$ glucose) without RANKL (no treatment [NT] control), N-Glc with RANKL, normoglycemic conditions with high osmotic pressure (Man, 5.5 mM glucose and 10.0 mM mannitol) and RANKL, and hyperglycemic conditions (H-Glc, 15.5 mM glucose) with RANKL. The number of TRAP-positive multinuclear cells was counted in each well. (C) The effect of high-glucose treatment on Nfatc1 expression levels in RAW264.7 cells. The cells were cultured under hyperglycemic conditions for $3 \mathrm{~d}$, and protein expression in the cell lysates was analyzed by Western blotting, with the relative expression levels shown. (D) Mmp9 and (E) Ctsk mRNA expression levels in RAW264.7 cells after stimulation. These expression levels are shown relative to Gapdh expression. Values are presented as means \pm SD for at least three replicates. ${ }^{* *} p<0.01$. Relative expression levels for Western blotting were quantified by using the ImageJ program.

Differences for Fig. 3B were analyzed with two-way analysis of variance (ANOVA) with repeated measures, followed by Tukey's multiple comparison test. Other statistical analysis was performed with one-way ANOVA with repeated measures, followed by Tukey's multiple comparison test. $p$ values $<0.05$ were accepted as statistically significant.

\section{RESULTS}

High glucose treatment suppresses RANKL-induced OCL differentiation

To examine the effect of high glucose treatment on cell viability during RANKL stimulation, RAW264.7 macrophages were treated with $15.5 \mathrm{~mm}$ high glucose 
A
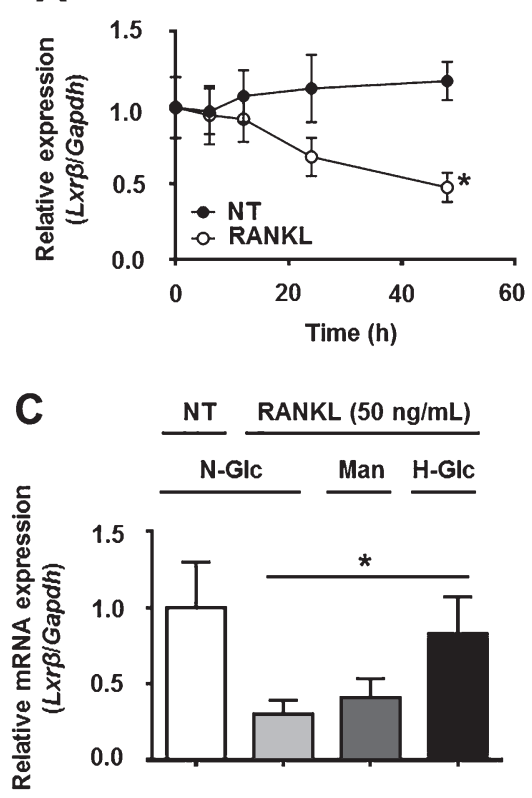

B

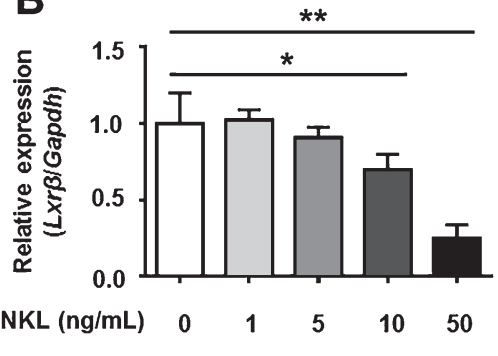

D

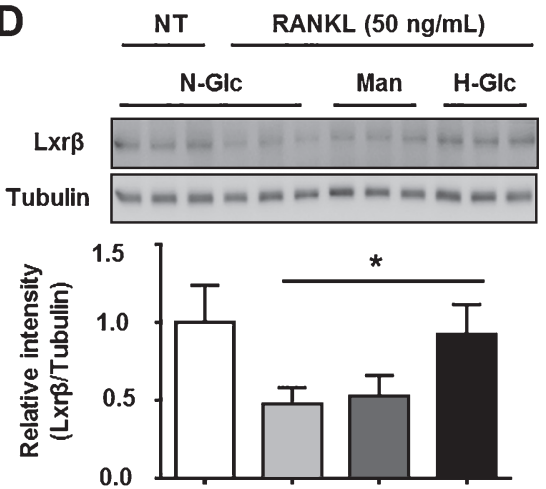

Fig. 2. High-glucose treatment increases mRNA and protein expression of Lxr $\beta$ in RANKL-induced osteoclastogenesis. Time-dependent (A) and dose-dependent (B) $\operatorname{Lxr} \beta$ gene transcription in RAW264.7 cells treated with RANKL. Lxr $\beta$ mRNA (C) and protein (D) expression levels in RAW264.7 cells after stimulation. These expression levels are shown relative to Gapdh and Tubulin expression. RAW264.7 cells were cultured for $2 \mathrm{~d}$ under normoglycemic conditions (N-Glc, $5.5 \mathrm{mM}$ glucose) without RANKL (no treatment [NT] control), N-Glc with RANKL, normoglycemic conditions with high osmotic pressure (Man, $5.5 \mathrm{~mm}$ glucose and $10.0 \mathrm{~mm}$ mannitol) and RANKL, and hyperglycemic conditions (H-Glc, $15.5 \mathrm{~mm}$ glucose) with RANKL. Values are presented as means \pm SD for at least three replicates. ${ }^{*} p<0.05,{ }^{* *} p<0.01$. Relative expression levels for Western blotting were quantified by using the ImageJ program.

in the presence of RANKL. Cell viability, numbers of cell recovery, total protein and RNA recovery were then evaluated. As shown in Table 1, high glucose treatment did not affect cell viability or numbers of cell after $2 \mathrm{~d}$. On the other hand, RANKL treatment significantly increased the RNA recovery, which was suppressed by high glucose treatment but not significantly. The results of cell viability, numbers of cell recovery, total protein and RNA recovery in BMMs show similar findings to those for RAW264.7 cells (data not shown).

Next, we performed enzymatic TRAP staining in primary macrophage cells to examine the effect of hyperglycemia on osteoclastogenesis. OCLs, defined as TRAP-positive multinucleated cells stained purple-red, were observed following RANKL treatment (Fig. 1A). High glucose suppressed RANKL-induced OCL differentiation (104.1 \pm 11.0 vs. $3.1 \pm 1.2$ cells/well, $p<0.01$ ). Although mannitol also suppressed osteoclastogenesis, the inhibitory effect was lower than for high-glucose treatment. As shown in Fig. 1B, we confirmed that high-glucose treatment significantly decreased the number of TRAP-positive multinuclear cells compared with normoglycemic conditions in RAW264.7 cells (130.4 \pm 25.8 vs. $17.4 \pm 6.8$ cells/well, $p<0.01)$. To further examine the effect of high glucose on osteoclastogenesis, protein expression levels of NFATc1, a master regulator of RANKL-induced OCL differentiation (20), were examined. The expression levels were significantly suppressed by high glucose compared with normal glu-

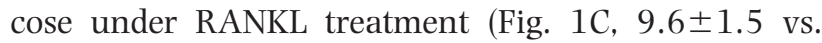
$5.2 \pm 1.1, p<0.01$ ), while mannitol treatment did not affect expression. NFATc1 binds directly to the promoter regions of its target genes, such as MMP9 and cathepsin K (Ctsk) (21), and we therefore investigated Mmp9 and Ctsk gene expression. High glucose also significantly suppressed RANKL-induced Mmp9 and Ctsk expression and secretion compared with normal glucose (Fig. 1D and E, $25.5 \pm 7.3$ vs. $11.3 \pm 1.9, p<0.01$; and $18.0 \pm 5.8$ vs. $7.5 \pm 2.9, p<0.05)$. These results indicate that high-glucose treatment suppresses RANKL-induced osteoclastogenesis.

High glucose increases Lxr $\beta$ expression levels in RANKLinduced OCL differentiation

To examine the mechanism by which high glucose suppresses osteoclastogenesis, we next measured $\operatorname{Lxr} \beta$ mRNA expression levels. As shown in Fig. 2A, Lxr $\beta$ mRNA expression level was time-dependently decreased by RANKL treatment. The expression of $\operatorname{Lxr} \beta$ mRNA in the cells treated with RANKL was also dose-dependently decreased at $1-50 \mathrm{ng} / \mathrm{mL}$ at $2 \mathrm{~d}$ and was significantly suppressed by 10 and $50 \mathrm{ng} / \mathrm{mL}$ RANKL treatment (Fig. $2 \mathrm{~B}, 1.0 \pm 0.2$ vs. $0.7 \pm 0.1$ and $1.0 \pm 0.2$ vs. $0.2 \pm 0.1$, respectively, $p<0.01)$. High-glucose treatment significantly restored the RANKL-induced suppression of Lxr $\beta$ mRNA expression (Fig. 2C, $0.3 \pm 0.1$ vs. $0.8 \pm 0.3$, $p<0.05)$, while mannitol treatment showed no signifi- 
A
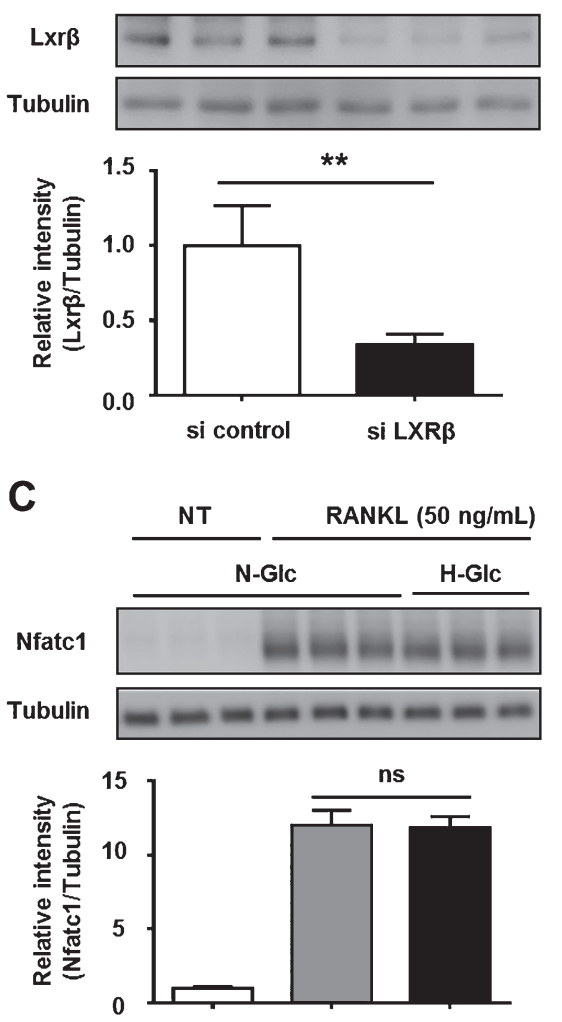

B

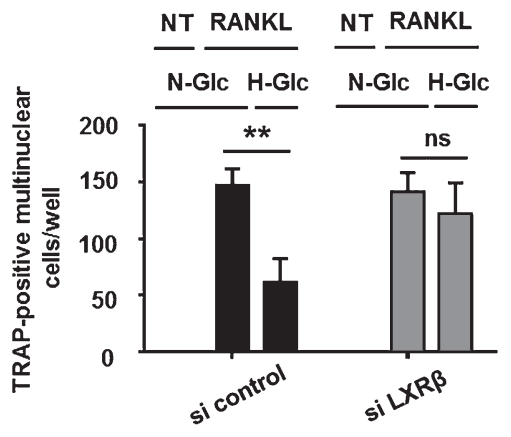

Fig. 3. LXR $\beta$ expression is needed for suppression of RANKL-induced osteoclastogenesis by high glucose. (A) LXR $\beta$ protein expression after LXR $\beta$ siRNA knock down. (B) The numbers of TRAP-positive cells in RAW264.7 cells at $5 \mathrm{~d}$ after transfection with control siRNA or LXR $\beta$ siRNA. (C) Nfatc1 protein expression levels in RAW264.7 cells at $3 \mathrm{~d}$ after transfection with LXR $\beta$ siRNA. The protein expression is shown relative to Tubulin expression. After a 4-h transfection, 3,000 or $5 \times 10^{5}$ cells were seeded in 96 - or 6 -well plates. The cells were then cultured under normoglycemic conditions (N-Glc, $5.5 \mathrm{~mm}$ glucose) without RANKL (NT), N-Glc with RANKL, and hyperglycemic conditions (H-Glc, $15.5 \mathrm{~mm}$ glucose) with RANKL. Values are presented as means \pm SD for at least three replicates. ${ }^{* *} p<0.01$. Relative expression levels for Western blotting were quantified by using the ImageJ program.

cant change. As shown in Fig. 2D, the same trend was observed in $\operatorname{Lxr} \beta$ protein expression. In the high-glucose treatment group, $\operatorname{Lxr} \beta$ protein expression was significantly higher than in the normal-glucose treatment group, according to densitometric analysis of Western blotting ( $0.4 \pm 0.1$ vs. $0.9 \pm 0.2, p<0.05)$.

The increase of LXR $\beta$ expression is needed for suppression of RANKL-induced osteoclastogenesis by high glucose

To further examine the effect of $\operatorname{LXR} \beta$ activation on OCLs, RAW264.7 cells were treated with LXR $\beta$ siRNA. As shown in Fig. 3A, LXR $\beta$ siRNA knocked down LXR $\beta$ protein expression to $29 \%$ compared with the control. In the cells treated with the siRNA control, highglucose treatment significantly suppressed the number of RANKL-induced TRAP-positive multinuclear cells

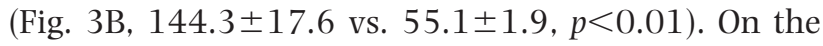
other hand, the RANKL-induced osteoclastogenesis was not significantly suppressed by high-glucose treatment in cells treated with LXR $\beta$ siRNA (138.3 \pm 21.7 vs. 115.2 \pm 33.5$)$. In this study, silencing of $\operatorname{LXR} \beta$ did not affect osteoclastogenesis under normoglycemic conditions. This may be explained by the temporary knock down of mRNA expression. Two-way ANOVA also showed interaction between si control with high-glucose and si LXR with high-glucose. High-glucose treatmentinduced suppression of NFATc1 protein expression levels (Fig. 1C) were restored by LXR $\beta$ siRNA treatment (Fig. $3 \mathrm{C}, 12.0 \pm 0.9$ vs. $11.7 \pm 0.7)$. These results suggest the involvement of $\operatorname{LXR} \beta$ in the mechanism by which high glucose suppresses osteoclastogenesis.

\section{DISCUSSION}

In the present study, we demonstrated that high glucose suppresses RANKL-induced osteoclastogenesis in vitro. Although mannitol also significantly suppressed the number of cells, the inhibitory effect was lower than for high-glucose treatment. Nfatc1 protein and Mmp9 and Ctsk gene expression levels were significantly suppressed by high glucose compared with RANKL treatment, while mannitol treatment did not affect expression. These results indicate that high-glucose treatment suppresses RANKL-induced osteoclastogenesis. The numbers of osteoclasts might be more reduced compared to the molecular markers Mmp9 and Ctsk. In this study, we determined the osteoclastic marker expression levels at single time point. Therefore, further studies at different time points might be needed to explain this difference between number of osteoclasts and the molecu- 
lar markers.

To investigate the mechanisms by which hyperglycemia suppresses osteoclastogenesis, we focused on the nuclear receptor $\mathrm{LXR} \beta$, which was once categorized as an orphan receptor but was later found to act as a glucose sensor (15). Our preliminary data in microarray suggest that high-glucose treatment up-regulates the mRNA expression levels in BMMs (data not shown). Interestingly, $\operatorname{Lxr} \beta$ expression was suppressed by RANKL stimulation under normal-glucose conditions but was not suppressed under high-glucose conditions. Deactivating this receptor by siRNA, on the other hand, canceled the suppressive effect of high glucose on osteoclastogenesis. These results suggest that hyperglycemiainduced up-regulation of LXR $\beta$ suppresses RANKLinduced osteoclastogenesis.

There are mainly two types of anti-osteoporotic agents. One is bone formation-stimulating agents, including fluoride and anabolic steroids. Another is antibone resorption agents, including estrogen, calcitonin, bisphosphonate, and raloxifene. Recently, Robertson Remen et al. reported that administration of the LXR agonist GW3965 protects lipopolysaccharide and/ or RANKL-induced inflammatory bone loss induced by inhibiting osteoclastogenesis in mice $(18,19)$. Our results also showed suppression of RANKL-induced osteoclastogenesis by GW3965 treatment (data not shown). Therefore, targeting $\operatorname{LXR} \beta$, which may act through the mimicry of hyperglycemia, is a potential therapeutic approach to treating osteoporosis.

The remaining challenges include a lack of understanding of the underlying pathway through which $\operatorname{LXR} \beta$ suppresses osteoclastogenesis. Many of the target genes of LXRs are involved in cholesterol metabolism regulation, including ATP binding cassette transporter isoforms A1 and G1 (22, 23), which are responsible for most of the cholesterol efflux in macrophage foam cells (24). Native low-density lipoprotein cholesterol increases OCL viability by suppressing spontaneous apoptosis (25). In addition, Jonarta et al. showed that high-density lipoprotein treatment partially reduced alveolar bone resorption in rats (26). Therefore, suppression of osteoclastogenesis by hyperglycemia may be dependent on $\mathrm{LXR} \beta$-induced activation of exogenous cholesterol composition, which may subsequently act on NF- $\kappa \mathrm{B}$. To further clarify the involvement of $\mathrm{NF} \kappa \mathrm{B}$ in LXR $\beta$-induced suppression of osteoclastogenesis, further studies at the different stages of osteoclastogenesis are probably warranted.

Generally, DM is an inflammatory disease. Although osteoclast differentiation is promoted by inflammatory response, bone mineral density is increased in type 2 DM patients, but not type $1 \mathrm{DM}$. The inhibitory effect of osteoclastogenesis by high glucose could explain this pathological condition in type $2 \mathrm{DM}$. On the other hand, Ferron et al. found that insulin signaling in osteoblasts integrates bone remodeling and energy metabolism (27). Therefore, the pathological condition of type 2 DM might not be sufficient to explain the relationship between hyperperglycemia and OCL.
The difference in glucose transportation between type 1 (insulin depletion) and 2 (insulin resistance) DM could explain this discrepancy. It is reported that glucose is a principal energy source for OCL differentiation (7). Indo et al. showed that during osteoclastogenesis the expression of both glucose transporter 1 (Glut1) and glycolytic genes is increased (5). There are some reports that hyperglycemia decreases glucose transporter expression levels in vitro $(28,29)$. Therefore, LXR $\beta$ activation may suppress osteoclastogenesis by downregulating Glut1 expression levels and subsequent TCA cycle and oxidative phosphorylation in mitochondria in RAW264.7 cells. However, most patients with type 2 diabetes are normo- or hyperinsulinemic. Since bone cells are insulin-sensitive, glucose homeostasis alone might not explain the mechanism by which hyperglycemia suppresses osteoclastogenesis.

In conclusion, high glucose inhibits RANKL-induced osteoclastogenesis, possibly by up-regulating $\mathrm{LXR} \beta$. Although further validation of the underlying pathway is necessary, targeting $\operatorname{LXR} \beta$ is a potential therapeutic approach to treating osteoporosis.

\section{Acknowledgments}

This work was supported by the American Heart Association project on the role of osteoclast-like cells in abdominal aortic aneurysm formation.

\section{REFERENCES}

1) Väänänen HK, Laitala-Leinonen T. 2008. Osteoclast lineage and function. Arch Biochem Biophys 473: 132-138.

2) Roy B. 2013. Biomolecular basis of the role of diabetes mellitus in osteoporosis and bone fractures. World J Diabetes 4: 101-113.

3) Kobayashi K, Takahashi N, Jimi E, Udagawa N, Takami M, Kotake S, Nakagawa N, Kinosaki M, Yamaguchi K, Shima N, Yasuda H, Morinaga T, Higashio K, Martin TJ, Suda T. 2000. Tumor necrosis factor alpha stimulates osteoclast differentiation by a mechanism independent of the ODF/RANKL-RANK interaction. J Exp Med 191: 275-286.

4) Cornish J, Callon KE, Bava U, Kamona SA, Cooper GJ, Reid IR. 2001. Effects of calcitonin, amylin, and calcitonin gene-related peptide on osteoclast development. Bone 29: 162-168.

5) Indo Y, Takeshita S, Ishii KA, Hoshii T, Aburatani H, Hirao A, Ikeda K. 2013. Metabolic regulation of osteoclast differentiation and function. J Bone Miner Res $\mathbf{2 8}$ : 2392-2399.

6) Williams JP, Blair HC, McKenna MA, Jordan SE, McDonald JM. 1996. Regulation of avian osteoclastic $\mathrm{H}+$-ATPase and bone resorption by tamoxifen and calmodulin antagonists. Effects independent of steroid receptors. J Biol Chem 271: 12488-12495.

7) Williams JP, Blair HC, McDonald JM, McKenna MA, Jordan SE, Williford J, Hardy RW. 1997. Regulation of osteoclastic bone resorption by glucose. Biochem Biophys Res Commun 235: 646-651.

8) Kim JM, Jeong D, Kang HK, Jung SY, Kang SS, Min BM. 2007. Osteoclast precursors display dynamic metabolic shifts toward accelerated glucose metabolism at an early stage of RANKL-stimulated osteoclast differentiation. Cell Physiol Biochem 20: 935-946. 
9) Wittrant Y, Gorin Y, Woodruff K, Horn D, Abboud HE, Mohan S, Abboud-Werner SL. 2008. High d(+)glucose concentration inhibits RANKL-induced osteoclastogenesis. Bone 42: 1122-1130.

10) Krinsley JS. 2003. Association between hyperglycemia and increased hospital mortality in a heterogeneous population of critically ill patients. Mayo Clin Proc 78: 1471-1478.

11) Vestergaard P. 2007. Discrepancies in bone mineral density and fracture risk in patients with type 1 and type 2 diabetes-a meta-analysis. Osteoporos Int 18: 427-444.

12) Leidig-Bruckner G, Ziegler R. 2001. Diabetes mellitus a risk for osteoporosis? Exp Clin Endocrinol Diabetes 109(Suppl 2): S493-514.

13) Catalfamo DL, Calderon NL, Harden SW, Sorenson HL, Neiva KG, Wallet SM. 2013. Augmented LPS responsiveness in type 1 diabetes-derived osteoclasts. J Cell Physiol 228: 349-361.

14) Catalfamo DL, Britten TM, Storch DL, Calderon NL, Sorenson HL, Wallet SM. 2013. Hyperglycemia induced and intrinsic alterations in type 2 diabetes-derived osteoclast function. Oral Dis 19: 303-312.

15) Mitro N, Mak PA, Vargas L, Godio C, Hampton E, Molteni V, Kreusch A, Saez E. 2007. The nuclear receptor LXR is a glucose sensor. Nature 445: 219-223.

16) Spillmann F, Van Linthout S, Miteva K, Lorenz M, Stangl V, Schultheiss HP, Tschöpe C. 2014. LXR agonism improves TNF-alpha-induced endothelial dysfunction in the absence of its cholesterol-modulating effects. Atherosclerosis 232: 1-9.

17) Wang Y, Li C, Cheng K, Zhang R, Narsinh K, Li S, Li X, Qin X, Zhang R, Li C, Su T, Chen J, Cao F. 2014. Activation of liver $\mathrm{X}$ receptor improves viability of adiposederived mesenchymal stem cells to attenuate myocardial ischemia injury through TLR4/NF-kappaB and Keap-1/ Nrf-2 signaling pathways. Antioxid Redox Signal 21: 2543-2557.

18) Robertson Remen KM, Lerner UH, Gustafsson JA, Andersson G. 2013. Activation of the liver X receptorbeta potently inhibits osteoclastogenesis from lipopolysaccharide-exposed bone marrow-derived macrophages. J Leukoc Biol 93: 71-82.
19) Remen KM, Henning P, Lerner UH, Gustafsson JÅ, Andersson G. 2011. Activation of liver X receptor (LXR) inhibits receptor activator of nuclear factor $\kappa \mathrm{B}$ ligand (RANKL)-induced osteoclast differentiation in an LXR $\beta$ dependent mechanism. J Biol Chem 286: 33084-33094.

20) Nakashima T, Takayanagi H. 2009. Osteoclasts and the immune system. J Bone Miner Metab 27: 519-529.

21) Song I, Kim JH, Kim K, Jin HM, Youn BU, Kim N. 2009. Regulatory mechanism of NFATc1 in RANKL-induced osteoclast activation. FEBS Lett 583: 2435-2440.

22) Edwards PA, Kennedy MA, Mak PA. 2002. LXRs; oxysterol-activated nuclear receptors that regulate genes controlling lipid homeostasis. Vascul Pharmacol 38: 249-256.

23) Bensinger SJ, Tontonoz P. 2008. Integration of metabolism and inflammation by lipid-activated nuclear receptors. Nature 454: 470-477.

24) Yvan-Charvet L, Wang N, Tall AR. 2010. Role of HDL, ABCA1, and ABCG1 transporters in cholesterol efflux and immune responses. Arterioscler Thromb Vasc Biol 30: 139-143.

25) Luegmayr E, Glantschnig H, Wesolowski GA, Gentile MA, Fisher JE, Rodan GA, Reszka AA. 2004. Osteoclast formation, survival and morphology are highly dependent on exogenous cholesterol/lipoproteins. Cell Death Differ 11 (Suppl 1): 108-118.

26) Jonarta AL, Pudyani PS, Sosroseno W. 2002. Effect of high-density lipoprotein on lipopolysaccharide-induced alveolar bone resorption in rats. Oral Dis 8: 261-267.

27) Ferron M, Wei J, Yoshizawa T, Del Fattore A, DePinho RA, Teti A, Ducy P, Karsenty G. 2010. Insulin signaling in osteoblasts integrates bone remodeling and energy metabolism. Cell 142: 296-308.

28) Oleszczak B, Szablewski L, Pliszka M. 2012. The effect of hyperglycemia and hypoglycemia on glucose transport and expression of glucose transporters in human lymphocytes B and T: an in vitro study. Diabetes Res Clin Pract 96: 170-178.

29) Kim DI, Lim SK, Park MJ, Han HJ, Kim GY, Park SH. 2007. The involvement of phosphatidylinositol 3-kinase/Akt signaling in high glucose-induced downregulation of GLUT-1 expression in ARPE cells. Life Sci 80: 626-632. 